\title{
Research on energy structure changes in the future --taking four states on the U.S.-Mexico border as examples

\author{
Zhedong $\mathrm{Hu}$ *
}

North China Electric Power University (Baoding), Hebei, China.

*2158827802@qq.com

\begin{abstract}
The change of energy structure depends on the change of production structure and consumption structure. This paper evaluates the changes of energy structure in the future by establishing the quadratic exponential smoothing model (QES) based on Prediction of time series (TSP). Firstly, according to extractable historical data from 1969 to 2009 in four states on the U.S.Mexico border (California (CA), Arizona (AZ), New Mexico (NM) and Texas (TX)), we can gain line charts of energy production and consumption output to show the dynamic changes in the four states. Then, through provided charts, the energy structure changes in the future is obtained by using QES model.
\end{abstract}

Keywords: Change of energy structure, QES model, Prediction of time series, CA, AZ, NM, T X.

\section{The Trend of Energy Production Changes}

In order to grasp the trend of energy production changes of four states, we used the following line charts to analyze.
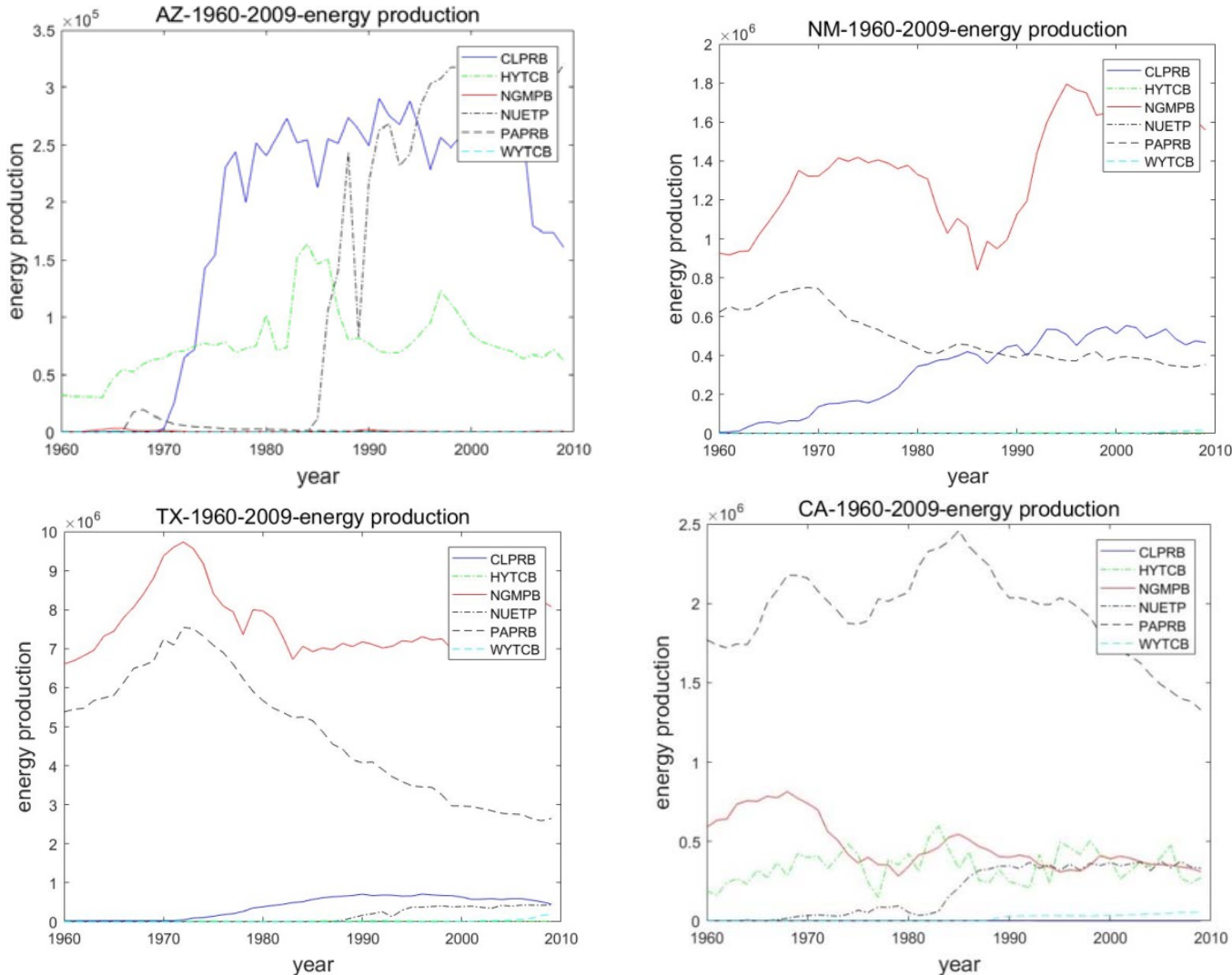

Fig. 1 Energy Production Changes of Four States

According to the above figures, the trends of change in the four states also vary with each other. For Arizona, CLPRB begins to surge in 1970, stabilize in 1980, and continue to rise and fall after that. However, the supply of NUETB lags behind that of CLPRB, though the growth pattern is the same. The trend of other energy sources is basically stable. In California, PAPRB has been in the main position all the time, and NGMPB is much larger than that of the rest of the energy. In 1987, the 
NGMPB of New Mexico is at a low point. And NGMPB and PAPRB, which have been a large share of the energy supply, both with peaks in 1970. But NGMPB has been relatively stable in recent years, and PAPRB has declined. Then, the rest production types of the total are not too volatile.

\section{The Trend of Energy Consumption Changes}
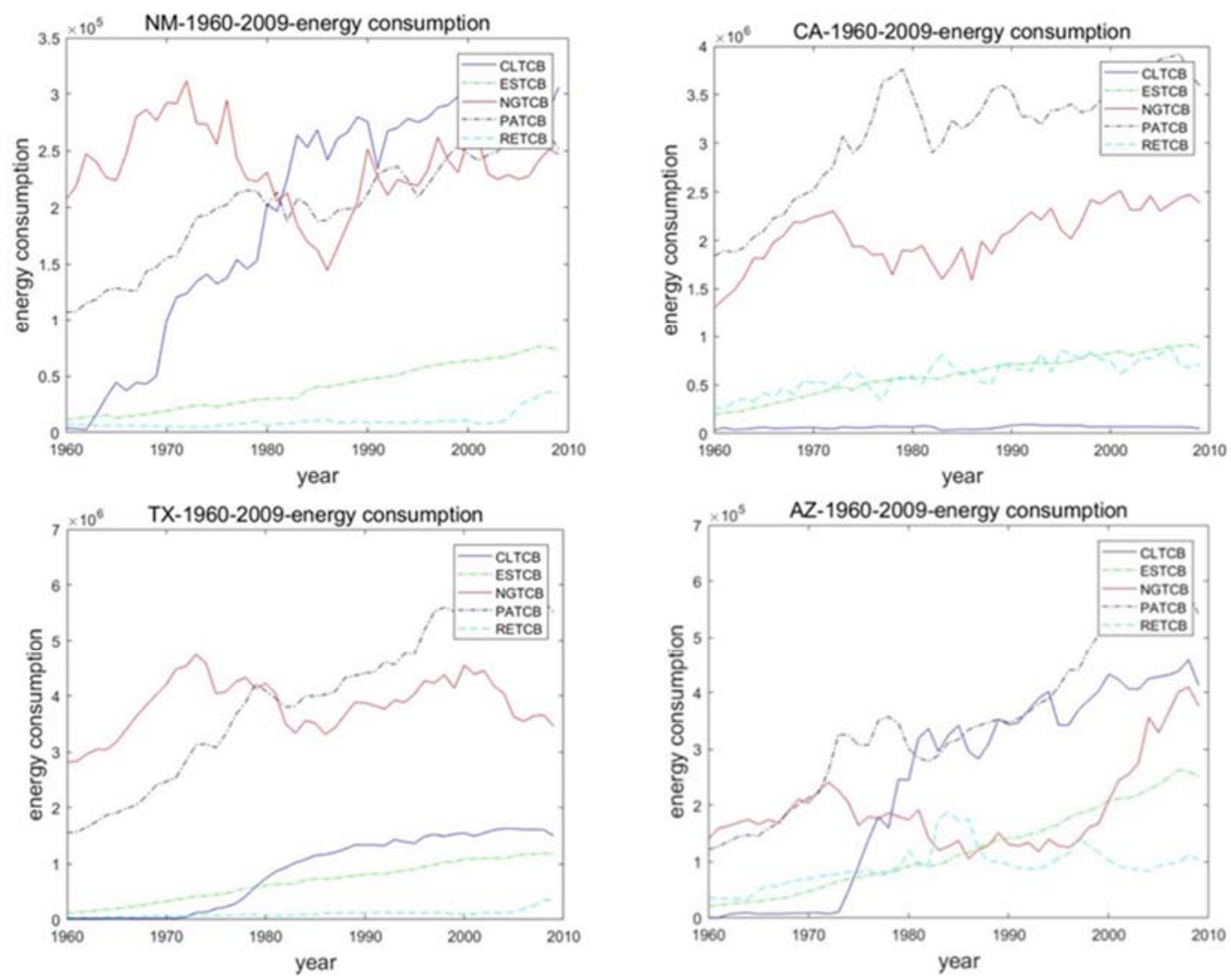

Fig. 2 The Changes of Energy Consumption from 1969 to 2010

From the above line charts, it is clear that natural gas total consumption was roughly in a balanced changing pattern except for Arizona, for Arizona was in the growth pattern. What's more, Arizona and New Mexico increased sharply. Texas was in a slight growth model, and California had almost no change.

\section{The Change of Energy Structure in the Future}

In order to infer the possibility of future changes in the energy structure of the four states, as well as the trends and laws of changes, we have used the time series prediction method, based on the historical data from 1960 to 2009 , to carry out statistical analysis, properly process the data and also forecast the trend of changes in 2025 and 2050.

Because the impact of historical data on future values decreases with the increase of time intervals, we need to take the weighted average of observed values of each period in time order as the predicted value, so as to more practical. Additionally, we need to choose the quadratic exponential smoothing method here.

\subsection{Prediction Model}

First we let the time series be $\mathrm{y}_{1}, \mathrm{y}_{2} \cdots \mathrm{y}_{\mathrm{n}}$, and $\alpha$ is the weighting coefficient, which satisfies the range of 0 to $1(0<\alpha<1)$. Considering the single exponential smoothing method has obvious lag error when the time series appear straight line trend, we must correct it, and then make quadratic exponential smoothing, and also establish the linear prediction model by using the law of deviation 
of lag error. We have selected the data from 1969 to 2009 as the initial values and established a quadratic exponential smoothing model.

The quadratic exponential smoothing formula is

$$
\left\{\begin{array}{c}
S_{t}^{(1)}=\alpha y_{t}+(1-\alpha) S_{t-1}^{(1)} \\
S_{t}^{(2)}=\alpha S_{t}^{(1)}+(1-\alpha) S_{t-1}^{(2)}
\end{array}\right.
$$

In this formula, $S_{t}^{(1)}$ is the smoothing value of the single exponential smoothing and $S_{t}^{(2)}$ is the smoothed value of the quadratic exponential smoothing. When the time series $\left\{y_{t}\right\}$ has a straightline trend from a certain period, we can predict it by a straight-line trend model as follows:

$$
\begin{gathered}
\hat{y}_{t+m}=a_{t}+b_{t} m, m=1,2, \cdots, \\
\left\{\begin{array}{l}
a_{t}=2 S_{t}^{(1)}-S_{t}^{(2)} \\
b_{t} \frac{\alpha}{1-\alpha} S_{t}^{(1)}-S_{t}^{(2)}
\end{array}\right.
\end{gathered}
$$

Based on the analysis and framework of the model, we have simulated the energy profile of the four states from 1960 to 2050 by using matlab to calculate, which is shown in the following figures. First part is four figures of energy consumption prediction.
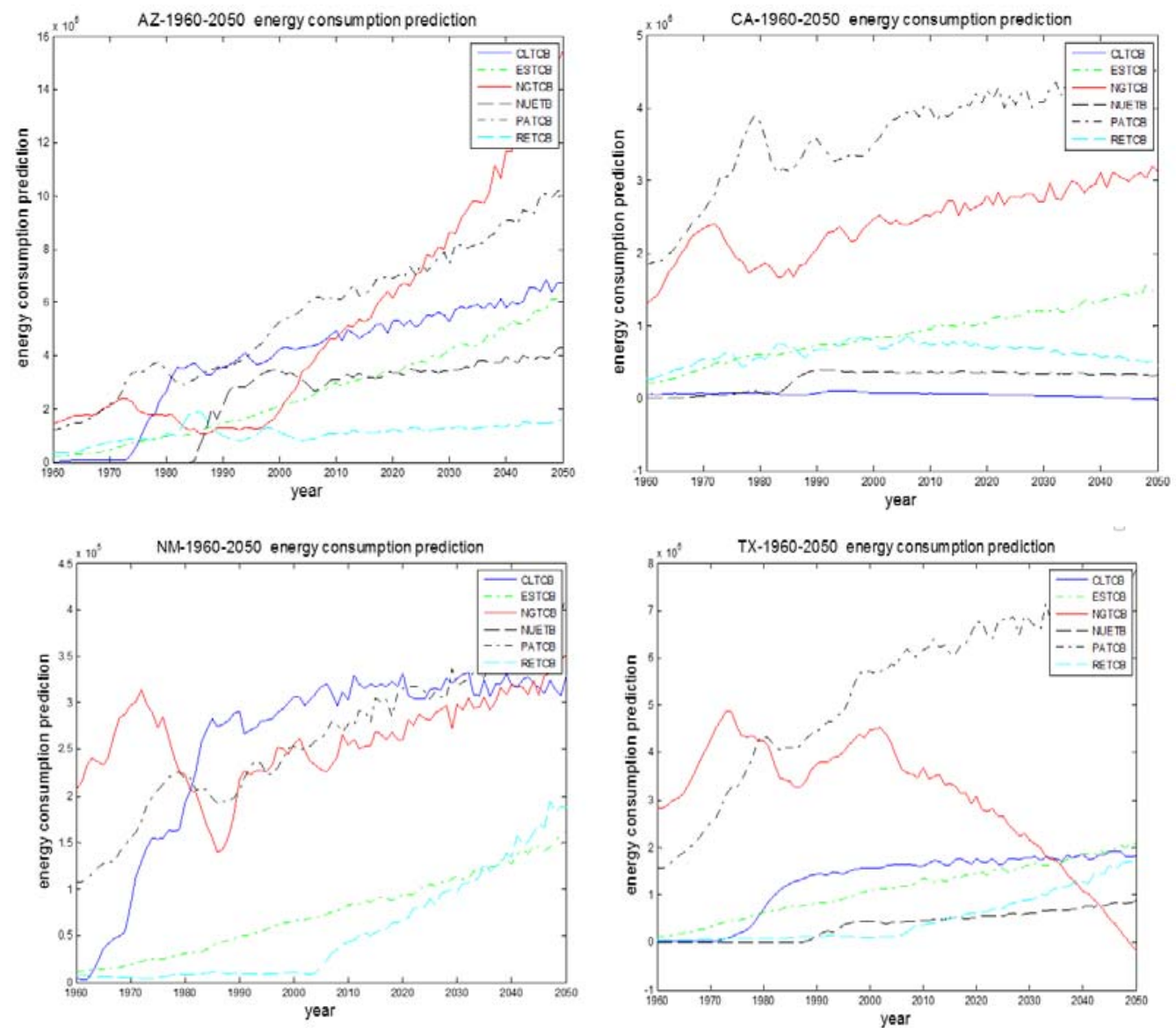

Fig. 3 Energy Consumption Prediction

As can be seen from the figures, different states have different trends of changes. Prior to 2025, there is a small increase in the number of the six variables of Arizona. However, the variables in CA are stationary. As for New Mexico, the variables are also rolling up in fluctuation and NETCB is at a low point in 1988. Then, NETCB also hits the lowest point in 1988 in Texas. On the contrary, in the same year, CLTCB is in a state of steep growth in Texas. After 1980, the other variables all increase mildly in Texas.

During the period from 2025 to 2050, all variables of Arizona are in the growth pattern, but the growth rate of NGTCB is much faster than the other variables. For California, CLTCB and NGTCB are in a fluctuating growth pattern while the other variables were stable. Each variable in New Mexico 
is also in the growth mode. For Texas, all variables except NGTCB were generally increasing. It should be pointed out that the changing trend of NGTCB is the most sensitive, which is in a growth state in Arizona, California and New Mexico. In the opposite, it decreases sharply in Texas. The reasons can be summed up in the following three points:

- The NGTCB in Texas is so sensitive that we could hardly estimate its changing trend.

- The quadratic exponential smoothing method is hindered by the condition when analyzing the NGTCB of Texas, which leads to the inevitable deviation problem.

- Time series could not reflect their internal relations.

Then, the second figures are about prediction of energy production of the four states.
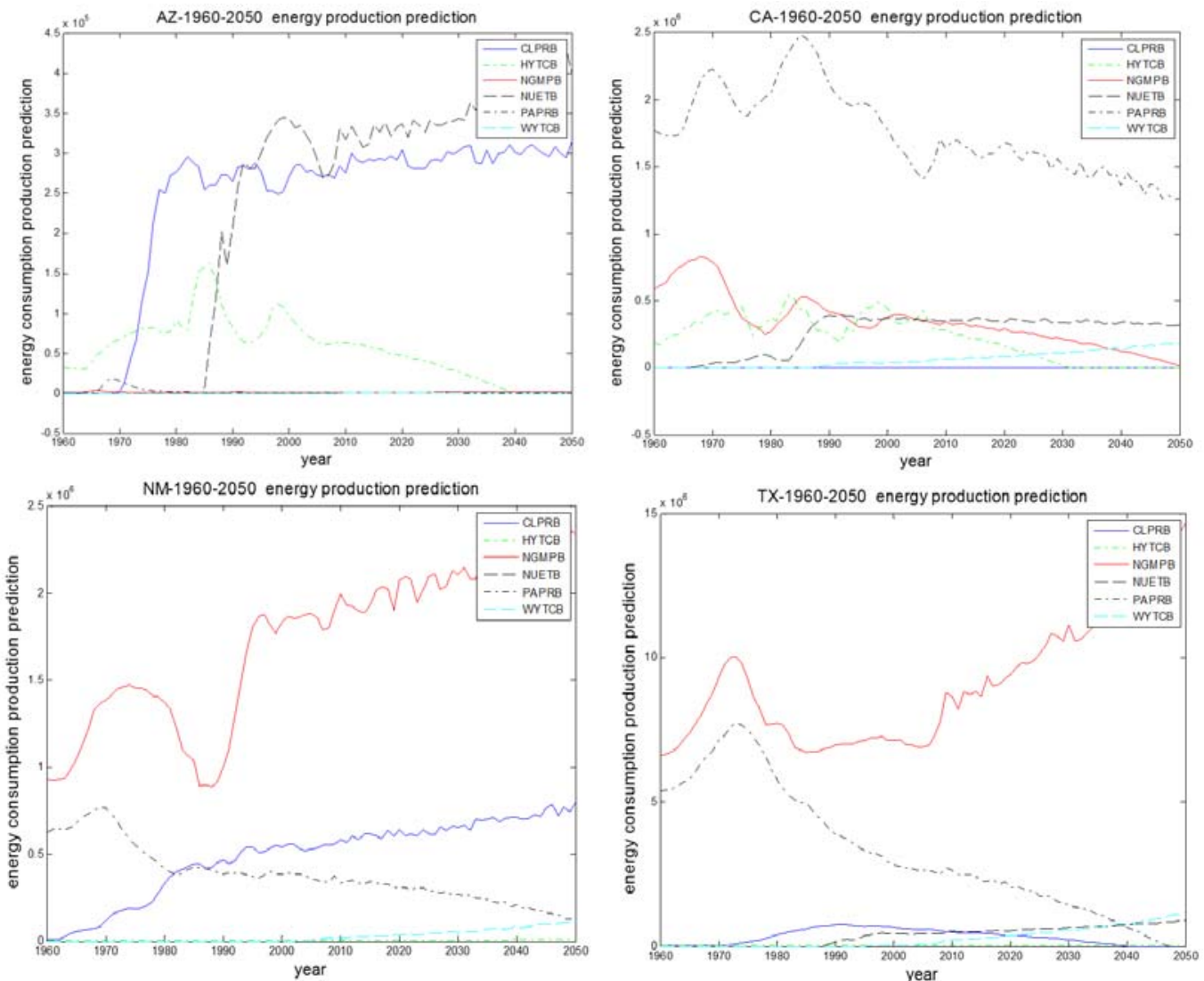

Fig. 4. Prediction of Energy Production of the Four States

We can see from the above figures that, the trends of changes in the four states is different between the period before and after 2025. For Arizona before 2025, the six variables first increase substantially and then the trend is dithering on 1980 and 1890. What's more, the changing trend of WYTCB, PAPRBCA and CLPRB all remained steady. As for New Mexico, there is an obvious low peak of NGPMB in 1990, while the other variables remain almost unchanged. Lastly, there is a significant increase of NGMPB and PAPRB in1970.

Then, after 2050, the trend of NUETB in Arizona shoots up, while the trend of HYTCB drops gradually to 0 , with other variables in steady. Furthermore, the changing trend of six variables in California also tends to be stable. Moreover, the trend of NGMPB and NGMPB rises in New Mexico. At last, the NGMPB also climbs in Texas, while PAPRB tends to be 0 .

\section{Predicted Results}

The energy supply of Arizona will be dominated by NUETB and NUETB, so coal and nuclear power supply will be the main parts of the energy supply structure of the state. And additionally, the industrial structure will move towards nuclear and coal industries. The state will be promoted to a huge coal and nuclear power supply states, with the rest of the energy supply gradually shrinking. 
However, NGMPB will continue to increase significantly in the consumption sector, and the supply of natural gas will fall far short of its consumption, which will result in the passive structure of the state's natural gas. In addition, the consumption of each energy source is increasing, which will meet the principle of necessity for social development and economic progress.

PAPRB will become a mainstream industry in the coming decades in California, and its supply will cover the rest of the energy supply. The state's crude oil will become the mainstream industry, and the supply of nuclear energy will be stable. But its share is far lower than crude oil, and the state's energy mix will be strained by rising oil and gas consumption due to inadequate supplies.

The energy supply of New Mexico will mainly depend on NGMPB. Besides, the supply of natural gas is the characteristic supply to the state, and the supply of coal will increase by a small margin. Additionally, the interlaced supply of coal and natural gas is the development prospect of the state. Furthermore, the consumption amounts of coal, oil and natural gas are soaring. Gas and coal supplies largely meet the state's internal needs, and oil consumption needs to be replenished.

The abnormal growth of NGMPB in Texas is in sharp contrast to the sharp drop in PAPRB. In the coming decades, the supply structure of natural gas will cover a large area, while crude oil will be phased out in the energy structure. As a consequence, the state's energy structure is almost stable. The rapid consumption and abundant supply of natural gas promote the flow of social strata and make the society more closely connected.

\section{References}

[1]. The mathematical modeling algorithm and application [M]. Beijing: national defense industry press.2011.

[2]. Department of Energy and Climate Change. The UK low carbon transition plan[R]. UK, 2009.

[3]. Sun lingxiao, shi weidong. Construction and empirical analysis of the evaluation model of regional energy sustainable development in China [J]. Assets and industry,2008, 8:17-23.

[4]. Pleßmann G, Erdmannm M, Hlusiak M, et al. Global energy storage demand for a $100 \%$ renewable electricity supply [J]. Energy Procedia, 2014, 46:22-31.

[5]. Utama N A, Fathoni A M, Kristianto M A, et al. The End of Fossil Fuel Era:Supply-demand Measures through Energy Efficiency[J]. Procedia Environmental Sciences, 2014, 20: 40-45. 